\title{
La convivencia escolar en un grupo de segundo grado de primaria
}

\section{School coexistence in a second grade elementary school group}

\author{
Graciela Rojo Bojorques • Martina Vega Cueto - Teresa Jiménez Álvarez
}

Graciela Rojo Bojorques. Escuela Normal Rural Ricardo Flores Magón, Saucillo, Chihuahua, México. Diplomado en línea para la educación de los derechos humanos, $\mathrm{CNDH}$, asistencia a congresos de educación especial; participante en la vertiente de cuentos "Don Quijote nos invita a leer" y en el Programa Institucional de Lectura convocado por el Departamento de Formación y Actualización Docente. Correo electrónico: rojob1328@gmail.com. ORCID: https:// orcid.org/0000-0003-2608-230X.

Martina Vega Cueto. Escuela Normal Rural Ricardo Flores Magón, Saucillo, Chihuahua, México. Es profesora-investigadora e integrante del Cuerpo Académico en Consolidación ENRRFM-CAEC-3 "Formación ética del profesorado". Perfil Proder desde el año 2015. Realizó estudios de Normal Básica en la licenciatura en Educación Primaria; licenciada en Educación Primaria en la UPN, maestra en Educación Campo Práctica Docente y doctora en Creatividad e Innovación Educativa por el Colegio Nueva Vizcaya. Asistencia y participación como ponente en congresos nacionales e internacionales. Correo electrónico: matty_vega_cueto@hotmail.com. ORCID: https://orcid.org/0000-0002-7017-3690.

Teresa Jiménez Álvarez. Escuela Normal Rural Ricardo Flores Magón, Saucillo, Chihuahua, México. Investigadora e integrante
Resumen

Para una buena convivencia, es fundamental que los valores se vivencien. El ambiente familiar influye de manera decisiva en la personalidad de sus integrantes, es un medio educativo al que se debe dedicar tiempo y esfuerzo, lo que el niño aprende en casa es reflejado en el contexto escolar. De ahí el interés en llevar a cabo la investigación educativa con el tema "la convivencia escolar en un grupo de segundo grado de primaria", que permita identificar los factores que interceden y repercuten en el aprendizaje de los alumnos. En el sustento teórico se dan a conocer las diversas aportaciones de estudiosos coadyuvantes a este tema de estudio. La metodología empleada fue bajo el paradigma interpretativo, enfoque cualitativo, método de etnografía; como técnicas se utilizaron la observación participante y la entrevista, se apoyó con los instrumentos del diario de campo y el cuestionario. Para la selección de participantes se tomó a los alumnos, padres de familia y docente del grupo escolar, lo que representó a la muestra no probabilística y de la cual se eligió el tipo por conveniencia. En los resultados se obtuvieron tres categorías: convivencia escolar, factores influyentes y aprendizaje. Los principales hallazgos encontrados son que por lo general existe una buena convivencia en el aula, pero también hay momentos de conflictos con algunos estudiantes, cuando ellos faltan a clases el ambiente es más armónico y se logran mayores aprendizajes. Se concluye que una educación basada en valores contribuye a la formación de mejores ciudadanos.

Palabras clave: Convivencia escolar, aprendizaje, estudiantes, factores sociales, valores.

Abstract
For a good coexistence, values must be lived. The family environment has a
decisive influence on the personality of its members, it is an educational environ-
ment to which time and effort must be devoted because what the child learns at
home is reflected in the school context. From there comes the interest to conduct
this educational research on the topic "School coexistence in a second-grade


del Cuerpo Académico en Consolidación ENRRFM-CAEC-3 "Formación ética del profesorado". Perfil Prodep desde el año 2015. Cuenta con estudios de licenciatura en Educación Preescolar en la ENRRFM, licenciatura en Educación por la UPN, licenciatura en Educación Media área Ciencias Sociales en la Escuela Normal Superior de La Laguna y maestría en Educación por el Tecnológico de Monterrey. Asistencia y participación como ponente en congresos nacionales e internacionales. Correo electrónico: teresajim28@gmail.com. ORCID: https:// orcid.org/0000-0002-4550-980X. elementary school group", which allows to identify the factors that intervene and affect the students' learning. In the theoretical background, the work of scholars who contribute to this subject of study is analyzed. The methodology used was under the interpretative paradigm, qualitative approach, ethnography method; the techniques used were participant observation and interview, supported with the instruments of field diary and questionnaire. For the selection of participants, students, parents, and teachers of the school group were selected, which represented the non-probabilistic sample and from which the type was chosen for convenience. Three categories were obtained in the results: school coexistence, influential factors, and learning. It was found that there is usually a good coexistence in the classroom, although there are also moments of conflict with some students, for example. When those students miss classes, the environment is more harmonious and greater learning is achieved. It is concluded that a values-based education contributes to the training of better citizens.

Keywords: School coexistence, learning, students, social factors, values.

\section{INTRODUCCIÓN}

El presente trabajo de investigación se realizó en un grupo de segundo grado en la Escuela Primaria Estatal Evolución Juvenil 2307, perteneciente a la Zona Escolar 87, con clave 08EPR01175D, ubicada en la comunidad del Kilómetro Noventa y Nueve, en el municipio de Rosales, Chihuahua. En la actualidad se tiene que en los centros escolares se presentan constantemente problemas, inequidad, discriminación y falta de valores, lo que conlleva a una inadecuada convivencia. La institución educativa ha de permitir que los estudiantes sean capaces de comprender, respetar y aceptar las diferentes formas de pensar, usos y costumbres que se poseen, además, que estén abiertos a descubrir e indagar ante la diversidad que se hace presente.

Para ello es indispensable que el docente se dé a la tarea de detectar los factores que pueden incidir en el aprendizaje del alumno, así mismo implementar estrategias para el fomento de valores, como trabajo colaborativo con los padres de familia para coadyuvar en los estudiantes; que sean capaces de aprender a convivir dentro de un ambiente armónico donde su participación sea interactiva con sus iguales, los docentes, la familia y la comunidad en que se desenvuelven. Por otro lado, también es necesario crear conciencia en las personas de que una desfavorable convivencia lleva a problemas, prejuicios y discriminación que afectan en el desarrollo integral de los niños. Por ello surgió como interés indagar: ¿Cuáles son los factores que influyen en la convivencia escolar para el aprendizaje de los alumnos?

En atención a la problemática existente, se hace necesario tomar en cuenta las bases legales que sustentan los derechos de las personas regidos por la Constitución Política de los Estados Unidos Mexicanos, con énfasis en el artículo tercero, el cual hace mención en cuanto a la educación otorgada a la nación (DOF, 2019), establece que "toda persona tiene derecho a la educación, el cual es un medio para adquirir, ac- 
tualizar, completar y ampliar sus conocimientos, capacidades, habilidades y aptitudes que le permitan alcanzar su desarrollo personal y profesional; como consecuencia de ello, contribuir a su bienestar, a la transformación y el mejoramiento de la sociedad de la que forma parte" (p. 1). Hace énfasis en que la educación no solo se debe basar en transmitir conocimientos y saberes, sino en desarrollar la integridad de los alumnos, enfocada en una convivencia pacífica, en la que el alumnado pueda aprender a relacionarse, expresarse, imaginar, crear y aceptar la diversidad cultural, todo esto con base en el respeto, por lo que aprender a convivir es una parte elemental de la educación. Para ello es importante que los maestros se mantengan en constante capacitación, de esta manera se permitirá lograr el objetivo principal de brindar una educación de calidad para formar estudiantes capaces de resolver problemas, desarrollar su creatividad y relacionarse de manera armónica ante la sociedad, así mismo fomentar en los alumnos interés por la práctica de valores y crear un ambiente apto para aprender. La SEP (2017) menciona que la principal tarea de la escuela es coadyuvar al desarrollo de la capacidad para aprender a aprender, es decir, aprender a pensar. Esto implica que el docente tiene el deber de generar estrategias para motivar y fomentar interés en cada alumno y desarrollar en ellos un pensamiento crítico.

Por consiguiente, para el estado del conocimiento, se analizaron diversas fuentes de indagaciones educativas relacionadas con la temática, de las cuales se eligieron cinco por ser las más afines al objeto de este estudio, para lo cual se retomaron los datos considerados relevantes por sus aportes e información al campo educativo. En la primera investigación, Navarrete (2017) señala que consistió en la implementación de un programa orientado al refuerzo de la práctica de valores morales, autoestima en el estudiantado, para propiciar la mejora de los aprendizajes. Se encontró que es importante entrelazar los conocimientos, actitudes y valores de una objetiva educación en las escuelas, en donde se dedica un tiempo mínimo a esta enseñanza, sobre distintos temas, incitando que los y las estudiantes no consigan mejorar sus conocimientos y actitudes. Además se considera que el espacio educativo para el logro de los objetivos puede continuar promoviendo iniciativas y programas que fortalezcan la convivencia escolar.

El trabajo de Caballero (2010) es enfocado en las prácticas educativas que fomentan la convivencia dentro de la educación. Se obtuvo que la convivencia siempre está presente, sin embargo, muchas de las veces no se realizan las estrategias adecuadas que ayuden a mejorar los conflictos que se presentan constantemente por la indisciplina, dado que los docentes tienen que estar más inmiscuidos para ejecutar, crear tácticas y métodos que consideren indispensables para tener una buena formación.

La indagatoria de Nicolás (2015) fue guiada al estudio de la situación de la convivencia escolar, en el cual se deja ver claro que en un ambiente escolar donde prevalecen las conductas agresivas afecta la convivencia entre alumnos y docentes, por ende, el desenvolvimiento y el rendimiento escolar de algunos estudiantes se ven afectados. Aunque es importante tomar en cuenta que los estudiantes de grado superior son más críticos, o tienen una percepción más negativa del clima en el centro educativo. 
En tanto, García y Ferreira (2005) trataron en su investigación sobre la convivencia escolar en las aulas como elemento básico para la calidad de la educación; consideraron la implementación de estrategias y técnicas para que los educandos aprendan a convivir mediante diversos valores. Se reconoce que se debe educar a los alumnos para que se responsabilicen en sus actividades escolares y familiares, es fundamental que los valores se fomenten desde la casa para que se puedan ver plasmados dentro del ámbito educativo, ya que la familia es el primer ejemplo para los niños.

En este mismo sentido, en el caso de Espinoza, Ojeda, Pinillo y Segura (2010), de la temática tratada acerca de la convivencia escolar en una escuela básica se derivó que existe la posibilidad de proponer un plan de trabajo para que la comunidad escolar pueda articularse de manera democrática y participativa, motivado y propiciado desde una visión que posiciona la gestión de la convivencia como una tarea ineludible, que debe partir desde un liderazgo directivo, efectivo y democrático, capaz de involucrar a toda la comunidad escolar en este desafío.

En relación al sustento teórico de esta investigación, se retomó a varios estudiosos en cuyas aportaciones se explicitan algunos de los conceptos principales relacionados con la convivencia escolar: de acuerdo con Rodino (2012), convivir conlleva a una conversación armoniosa, así como también colaborar ante la diversidad existente. Esto quiere decir que tener "un ambiente de paz y tranquilidad, es viable para interactuar, además, conocer otras culturas sin ningún conflicto, por lo que da un acceso a crear conocimientos y aprendizajes significativos por medio de intercambio de ideas y opiniones".

Trabajar de manera colaborativa es esencial para una buena convivencia. Se trata de aportar de manera conjunta, sin liderazgos, aceptando las opiniones y formas de apoyar e intervenir de cada individuo. El ambiente familiar influye de manera decisiva en la personalidad del niño, las relaciones entre los miembros de la casa determinan los modos de actuar de los niños, por eso la vida en la familia es un medio educativo al que es necesario dedicarle tiempo y esfuerzo. Ello se verá reflejado en el salón de clases, por lo que es básico e influye de cierta manera en la enseñanza formal; es un factor significativo para las oportunidades en la educación.

Por otro lado, para Ortega (2007) la convivencia es la suma de varios factores que intervienen en la conducta de las personas; respetarse entre ambas favorece la solución de problemas que se puedan presentar dentro del contexto. Se coincide con esta aportación: al convivir con un grupo de personas se tiene que respetar la diversidad que se presenta, debe llevarse a cabo una relación armoniosa que ayude a resolver problemas de manera conjunta y adquirir aprendizajes. El centro educativo es un espacio de interacción, convivir con otras personas es inevitable, se comienza con el núcleo familiar, y la escuela es donde pasan mayor tiempo, sin embargo, para muchos individuos es un problema, por ello es importante detectar los factores que intervienen y comprender qué es lo que resulta difícil para poder simpatizar de manera armónica. Una de las tareas de los docentes es apoyar e implementar estrategias para que los alumnos logren convivir. 
Según Mansilla (2014), "el alumno debe ser entendido como un ser humano que tiene la habilidad de socializar fácilmente, siendo protagonista de las interacciones sociales en las que se ve involucrado en su vida escolar y como ciudadano" (p. 23). Este autor le da la misma importancia a la transmisión de conocimientos y a la oportunidad de interactuar en una sociedad de manera respetuosa ante la diversidad de culturas y opiniones, sin dejar de lado el fomento de valores que favorezcan a una sana convivencia. Por ello es que el ambiente en casa influye de manera determinante en la conducta que presentan los alumnos dentro y fuera del aula, aquí mismo es donde se define la personalidad de cada individuo en torno a lo que observa y experimenta. Este autor hace énfasis en que si no se les inculca desde casa una vida basada en valores, los estudiantes difícilmente se apropiarán de estos. Es importante que se conozcan los factores que intervienen, para así mismo poder trabajar de forma conjunta entre alumno, docente y padres de familia.

En cambio, Mendive (2008) menciona que el fomento de valores es responsabilidad compartida entre la familia y la escuela, por lo que se deben inculcar y reforzar para que el niño se los apropie y pueda distinguir lo que es mejor para él. Por ello, es necesario que exista una comunicación permanente y el apoyo de la familia esté siempre presente, al igual que en la comunidad escolar es tarea del docente propiciar espacios donde se eviten enfrentamientos que perjudiquen la convivencia y el avance de los aprendizajes. El aprendizaje no solo se construye con base en los saberes del docente, sino que también se complementa con las vivencias que se adquieren conforme pasa el tiempo; en este caso, las primeras experiencias son en el núcleo familiar, por consiguiente, los alumnos comienzan a convivir con otro grupo de personas al ingresar al preescolar, es aquí cuando conocen y adquieren otro tipo de costumbres o maneras de actuar. El contexto del que se rodea es una parte fundamental, puesto que el ser humano aprende cuando interactúa y se relaciona.

Los valores son esenciales en la vida, son el pilar para la interacción y el bienestar de la sociedad, es por ello que la temática de este estudio se apoya en la teoría de Lanni (2005), que argumenta:

La convivencia escolar alude fundamentalmente al aprendizaje y para que sea posible, los intercambios entre todos los actores de la institución (alumnos, docentes y padres) que comparten la actividad en la escuela y que conforman esa red de vínculos interpersonales que se denomina convivencia, deben construirse cotidianamente, mantenerse y renovarse cada día, según determinados valores [p. 22].

Es decir que la convivencia es una parte esencial para llegar al objetivo principal que es el aprendizaje esperado, por lo que es importante construir un espacio de relación armónica acompañada de valores para que los alumnos aprendan a interactuar sin violencia y así mismo adquieran los conocimientos. Es aquí donde el docente tiene la labor de crear estrategias para que se vivencien valores que enriquezcan la relación en el aula. 


\section{Preguntas y objetivos de inVestigación}

Tener un panorama general del tema de estudio conllevó a un análisis en el cual surgieron preguntas de investigación: ¿Cómo es la convivencia escolar de los alumnos de segundo grado de primaria? ¿Cómo afecta el tipo de convivencia en el aprendizaje del grupo de segundo grado? ¿Qué factores intervienen en la convivencia escolar en el grupo de segundo grado? Los objetivos que se plantearon fueron: 1. Identificar la convivencia escolar de los alumnos de segundo grado de primaria, 2. Indagar cómo es que afecta la convivencia en el aprendizaje del grupo, y 3. Analizar los factores que intervienen en la convivencia escolar del grupo de segundo grado de primaria.

La relevancia de esta temática es que los alumnos aprendan a convivir en un entorno familiar, escolar y social, siendo un factor elemental para que se puedan adquirir los conocimientos requeridos, además del desarrollo integral de cada individuo que en un futuro forme parte de una sociedad humanista, capaz de aceptar la diversidad y resolver conflictos de la vida cotidiana. Se hace énfasis en la importancia de retomar una educación basada en valores, fomentándose desde casa y por consiguiente en los centros educativos.

Una mala convivencia afecta a los niños de distintas maneras, por ello esta investigación se enfoca en conocer los factores que intervienen y cómo obstaculizan un ambiente agradable. Se considera que la convivencia y el rendimiento escolar están enlazados, tener conocimiento de las consecuencias o repercusiones que se pueden presentar por una mala conducta es primordial para los docentes, de aquí pueden obtener respuestas a los problemas y lograr la implementación de estrategias para generar una adecuada armonía en el entorno, así como mejorar la educación.

\section{Metodología}

La metodología empleada fue bajo el paradigma interpretativo, según Sánchez (2013) "comprender y describir la realidad educativa a través del análisis profundo de las percepciones e interpretaciones de los sujetos intervinientes en las diversas situaciones de la investigación” (p. 96). Es decir, que la finalidad es llegar a una comprensión y un conocimiento profundo del grupo social a investigar, en el cual se debe tener la integración de los participantes. Es por ello que se hizo pertinente basarse en este paradigma porque trata de conocer y comprender las situaciones que se presentan, por lo que se relaciona con el tema de estudio enfocado en la convivencia escolar en el grupo. Se apoyó en el enfoque cualitativo señalan: Taylor y Bogdan (1987) se basa en la observación de los hechos, comprender lo que sucede coadyuva a realizar una descripción del acontecer y perspectiva de los sujetos estudiados dentro de un contexto. Esta investigación al ser de tipo cualitativa, permitió recolectar información del grupo investigado: alumnos, padres de familia y docente, así como poder comprender y encontrar los factores influyentes de manera directa en la conducta que presentaron los alumnos de segundo grado de la escuela primaria. 
Para la temática de este estudio, se eligió el método etnográfico, Creswell (2003) quien afirma que se utiliza para estudiar un grupo de individuos en su contexto natural, para recabar datos importantes de su comportamiento. Se consideró viable porque se detectaron las características, las interacciones interpersonales, los valores que practican en su vida, los factores intervienen en la convivencia escolar. Para ello, se tomaron en cuenta como técnicas: la observación participante, la cual permitió observar de cerca, al grupo investigado y la entrevista, que dio pauta para recoger datos con los informantes de alumnos y docente. Los instrumentos fueron: diario de campo en donde se registraron los acontecimientos del quehacer educativo y el cuestionario por ser el adecuado para la aplicación con los padres de familia. Cabe mencionar que fue la muestra no probabilística, de la cual se eligió el tipo de conveniencia por la oportunidad de la cercanía al grupo escolar estudiado. De acuerdo con el análisis de la información recabada de los diferentes instrumentos aplicados a los actores participantes aportaron interesantes datos empíricos para esta indagación.

Como resultados se obtuvieron tres categorías, convivencia, factores influyentes y aprendizaje. En la primera, se encontró entre participantes: alumnos, docente y padres de familia que en los hallazgos hay una relación significativa en la cual existe una convivencia sana dentro del aula, pero también que hay momentos en que se presentan conflictos y disgustos con los mismos niños de siempre, que hacen que las clases sean tediosas por tantos gritos y pleitos. Otro hallazgo fue, que hay algunos padres de familia, que no tienen la noción de los problemas que en ocasiones suelen ocurrir con sus hijos.

En este mismo sentido se obtuvo que en el diario de campo se hallaron registros semejantes con las respuestas de los informantes: "es un grupo de niños muy alegres, platicadores, respetuosos, empáticos, comparten sus cosas, ayudan a los compañeros que se les dificulta". Por lo tanto, se asume que la convivencia dentro del grupo escolar es buena, a excepción de aquellos niños que faltan al respeto a sus iguales como a los docentes, también cuando suelen hacer desorden e incitan a otros alumnos, lo que conlleva a una conducta negativa que afecta la disciplina de los estudiantes en el salón de clases.

Según Ortega (2007), "la convivencia encierra un bien común que es conveniente respetar, lo que favorece la espontánea resolución de conflictos” (p. 25). Al respecto, se observó en el aula que, aunque en ciertos momentos se presentaron problemas, buscaron las formas para resolverlos de la mejor manera, esto para la autora es parte vital para una buena convivencia.

Otro hallazgo del diario de campo es que la convivencia es totalmente sana y armoniosa cuando dos de los alumnos por alguna razón faltan a clases, en dichas ocasiones los demás niños se muestran atentos, trabajan en armonía, comentan lo tranquilo que se sienten sin gritos o llamadas de atención hacia ellos, juegan muy a gusto sin tener miedo de que los molesten o les quiten sus juguetes.

La segunda categoría se enfoca en los principales factores que influyen en una convivencia, en este caso son los valores que los alumnos conocen o les inculcan des- 
de casa, las reglas por las que se rigen y cómo es la convivencia en su familia. Según Martínez-Otero (2001), los problemas familiares tienen gran impacto en el desarrollo de los niños; entre dichos problemas, la falta de atención que reciben en casa, los métodos educativos utilizados, los permisos excesivos, la violencia, las expresiones verbales no adecuadas, son puntos clave para que al alumno se le dificulte convivir de manera pacífica con un grupo de personas en el contexto escolar.

Lo encontrado permitió conocer la influencia en el comportamiento de los niños, el hallazgo identificado es la falta de práctica de los valores, como lo son el respeto, la honestidad y la responsabilidad, así como también el interés, la motivación y el cuidado. En el diario de campo se hicieron anotaciones de ciertos días en los que los niños asistían desmotivados, desarreglados, sin tareas y con excusas de por qué no las realizaron, con pláticas de personas adultas e historias de lo que hicieron en días anteriores en la calle.

La tercera categoría corresponde al aprendizaje, si afecta o no el tipo de convivencia de los alumnos. En este sentido, Moreno y Soler (2006) indican que "la disrupción como una conducta que va contra la tarea educativa propiamente dicha, retarda el aprendizaje y hace que resulte más difícil” (p. 21). Tal y como lo mencionan estos autores, el tipo de convivencia que se da en el salón interviene en el aprendizaje de los alumnos; tener diversas interrupciones ocasionadas por mala conducta hace que los estudiantes se desconcentren y se desvíen de su objetivo.

Se obtuvo como hallazgo que a los alumnos bien portados no les afectó en su aprendizaje, en cambio hubo a quienes sí les perjudicó debido a distracciones, por jugar, platicar y molestar, además se les dificultó concentrarse en las clases. Esto es coincidente con lo registrado en el diario de campo: que la conducta de otros alumnos sí puede afectar a los niños que tienen interés en apropiarse de nuevos aprendizajes durante el desarrollo de las clases, fuera y dentro del aula.

Se concuerda con las y los autores, debido a que el grupo indagado en general presentó buena convivencia, pero la existencia de factores negativos como la mala conducta mostrada por algunos alumnos desarmonizó el ambiente escolar, lo que afectó el aprendizaje de varios compañeros; en cambio, los positivos favorecieron para vivenciar valores.

En la actualidad, los valores se dejan de lado, algunos de los alumnos difícilmente los han vivenciado en la familia, por lo tanto, es un arduo trabajo para los docentes. Pero es importante que, aunque sea difícil para los estudiantes adentrarse a un mundo de valores, los maestros propongan diversas estrategias para que se vivencien dentro y fuera del aula, esto les será significativo para una mejor convivencia tanto entre sus iguales como con las demás personas con que han de interactuar. Además es importante que el docente muestre interés y empatía por los niños; es el primer ejemplo para ellos en el salón de clases, su interés coadyuvará para que se motiven, se muestren felices, tranquilos y, sobre todo, sientan cariño, porque la realidad indica que hay quienes no reciben en casa este tipo de afectos que, en muchas ocasiones, es 
lo único que necesitan. El ser humano nace para interactuar y convivir con los demás; para llevar a cabo una convivencia favorable es necesaria la vivencia de los valores. La realización de este tema de estudio fue de impacto y relevancia con respecto a la educación de los niños, se consideraron los factores y aprendizaje del grupo.

\section{CONCLUSIONES}

Se concluye que una educación adecuada, basada en valores, en los contextos familiar y escolar ha de contribuir a la formación de buenos ciudadanos; al mismo tiempo, relacionarse con las personas y respetar las diversas opiniones coadyuva para poder resolver de manera conjunta los conflictos que se presenten en la vida diaria. Es importante que la convivencia entre los estudiantes sea favorable para que el aprendizaje sea significativo, pero para ello es esencial construir un espacio educativo donde prevalezca la armonía y que el alumnado interactúe sin violencia.

El proceso llevado a cabo en el tema de este estudio, así como la metodología y las aportaciones teóricas utilizadas, permitieron encontrar hallazgos significativos para fortalecer la convivencia tanto en el aula como en la familia, lo que derivó en el logro de los objetivos y preguntas de investigación que se plantearon. Se comprobó que existen diversos factores que influyen en la convivencia que se da dentro del salón de clases y que repercuten en el aprendizaje de los alumnos de segundo grado.

Los hallazgos principales encontrados son las coincidencias entre los informantes en que por lo general existe una buena convivencia, pero cuando se presentan conflictos dañan la armonía escolar; el descubrimiento más sobresaliente y sorprendente es que la convivencia cambia radicalmente cuando algunos alumnos no asisten a la escuela.

Existen varios factores que influyen de manera negativa en la convivencia dentro de un contexto. El ambiente en la familia es una parte primordial que se refleja ante la sociedad; de igual manera, la educación de principios en ocasiones es deficiente, por lo que algunos estudiantes ingresan a las instancias educativas sin vivenciar valores o reglas que son primordiales para una mejor sociedad.

La convivencia es inevitable entre dos o más personas, estará presente a lo largo de la vida de cada ser humano, por lo tanto, es necesario aprender a relacionarse con grupos de personas sin problema alguno, poder resolver conflictos de manera colaborativa, sin liderazgos, aceptar, respetar las opiniones y la diversidad de personalidad, sobre todo conocer y vivenciar los valores, pues estos facilitarán la convivencia con otros individuos de pensamientos distintos.

Este tema de investigación brinda aportaciones a la línea general del conocimiento dentro del ámbito educativo para mejorar la convivencia en el ambiente escolar, familiar y en otros entornos en que se desenvuelven los estudiantes. Así mismo se pretende que sea de gran utilidad para los docentes e investigadores, en contribución a la indagación sobre el tema de estudio. 


\section{REFERENCIAS}

Caballero G., M. (2010). Convivencia escolar; un estudio sobre buenas prácticas. Granada, España. Recuperado de: https://www.ugr.es/ revpaz/tesinas/rpc_n3_2010_dea5.pdf.

Creswell J., W. (2003). Research design: Qualitative, quantitative, and mixed methods approaches (2a. ed.). Thousand Oaks, California: Sage.

DOF [Diario Oficial de la Federación] (2019). Artículo tercero. Recuperado de: https://www.dof.gob. $\mathrm{mx} /$ nota_detalle.php?codigo $=5560457 \&$ fecha $=15 \% 2 \mathrm{~F} 05 \% 2 \mathrm{~F} 2019 \&$ print $=$ true.

Espinoza A., I., Ojeda S., P., Pinillo D., L., y Segura R., S. (2010). Convivencia escolar en una escuela básica. La Universidad Andrés Bello. Recuperado de: http://repositorio.unab.cl/xmlui/bitstream/handle/ ria/1487/Espinoza.

García, A., y Ferreira, G. (2005). La convivencia escolar en las aulas. International Journal of Developmental and Educational Psychology, 2(1), 163-183. Recuperado de: https://www.redalyc.org/ pdf/3498/349832309012.pdf.

Lanni, N. (2005). La convivencia escolar: una tarea necesaria, posible y compleja. Santiago de Chile.

Mansilla M., S. (2014). Rol del educadory del alumno en aulas de preprimaria de un colegio privado al trabajar según la teoría socio-constructivista. Guatemala: Campus central Guatemala de la Asunción.

Martínez-Otero, V. (2001). Convivencia escolar: problemas y soluciones. Revista Complutense de Educación, 12(1), 295-318. Recuperado de: https:// revistas.ucm.es/index.php/RCED/article/view/17762.

Mendive M., G. (2008). La escuela y sus desafíos. Declaración Universal de los Derechos Humanos.

Moreno, A., y Soler, M. P. (2006). La disrupción en las aulas: problemas y soluciones. España: Ministerio de Educación y Ciencia.

Navarrete C., S. (2017). La convivencia escolary su impacto en el proceso de enseñanza aprendizaje. La Universidad de El Salvador. Recuperado de: https://docplayer.es/91959321-Presentado-por-prof-sebastianmartir-navarrete-cornejo-prof-cristino-hernandez-romero.html.

Nicolás A., A. (2015). La convivencia escolar en los centros de educación secundaria de la región de Murcia: la voz del alumnado. España: Universidad de Murcia. https://digitum.um.es/digitum/ bitstream/10201/48163/1/Tesis $\% 20 \mathrm{La} \% 20$ convivencia $\% 20$ escolar $\% 20$ en $\% 201$ os $\% 20$ centros $\% 20$ de $\% 20$ Educaci $\%$ C3\%B3n $\% 20$ Secundaria $\% 20$ de $\% 201 \mathrm{a} \% 20$ Regi $\%$ C3 $\%$ B3n $\% 20 \mathrm{de} \% 20$ Murcia $\% 2 \mathrm{C} \% 20 \mathrm{la} \% 20 \mathrm{voz} \% 20 \mathrm{del} \% 20$ alumnado. $\% 20$ Ascensi $\% \mathrm{C} 3 \% \mathrm{~B} 3 \mathrm{n} \% 20 \mathrm{Nicol} \% \mathrm{C} 3 \% \mathrm{~A} 1 \mathrm{~s} \% 20$ Abenza..pdf.

Ortega R., R. (2007). La convivencia: un regalo de la cultura a la escuela. Catedrática de Psicología en la Universidad de Córdoba.

Rodino A., M. (2012). Recuperado de: https://www.redalyc.org/html/447/44732048005/.

Sánchez S., J. (2013). Paradigmas de investigación educativa: de las leyes subyacentes a la modernidad reflexiva. España: Universidad de Castilla La Mancha.

SEP [Secretaría de Educación Pública] (2017). Plan de estudios 2017. Aprendizajes clave. México.

Taylor S., J., y Bogdan, R. (1987). Introducción a los métodos cualitativos de investigación. Barcelona: Paidós.

Cómo citar este artículo:

Rojo Bojorques, G., Vega Cueto, M., y Jiménez Álvarez, T. (2020). La convivencia escolar en un grupo de segundo grado de primaria. RECIE. Revista Electrónica Científica de Investigación Educativa, 5(1), pp. $203-212$. doi: doi.org/10.33010/recie.v5i1.1090. 